\title{
Beismann, Dennis (2020): Eugen Kogon in der frühen Bundesrepublik. Ein öffentlicher Intellektueller zwischen Lehrstuhl und Fernsehstudio 1949-1969
}

\section{Berlin/Boston: De Gruyter Oldenbourg. 249 Seiten. 24,95€}

\author{
Eckhard Jesse
}

Angenommen: 23. September 2021 / Online publiziert: 8. Oktober 2021

(C) Der/die Autor(en) 2021

Das Bundesland Hessen erwies sich nach 1945 bei der Etablierung der hiesigen Politikwissenschaft als Vorreiter. Den ersten Lehrstuhl für Politikwissenschaft erhielt Wolfgang Abendroth 1950 in Marburg, Eugen Kogon (1903-1987) kam 1951 in Darmstadt nach einigem Hin und Her zum Zuge. Über einige Politikwissenschaftler aus der Gründergeneration sind Biografien erschienen, teils stärker auf das Leben (etwa von Simone Ladwig-Winters über Ernst Fraenkel), teils mehr auf das Werk gerichtet (etwa von Hans Lietzmann über Carl. J. Friedrich). Über Eugen Kogon gab es bisher keine Biografie. Auch diese geschichtswissenschaftliche Dissertation von Dennis Beismann, angefertigt an der Universität Kassel, ist keine umfassende Biografie, kommt einer solchen freilich nahe. Die Studie beschreibt, analysiert und bewertet das vielfältige Wirken Kogons in seinen zwei produktivsten Jahrzehnten, den 50er- und 60er-Jahre des vergangenen Säkulums, ist also weniger eine Werkbiografie.

In einem biografischen Prolog lässt Beismann die turbulente Zeit bis Ende der 1940er-Jahre Revue passieren: Kogons Studium der Nationalökonomie und der Soziologie in Wien mit seiner Dissertation (,Faschismus und Korporativstaat“) bei Othmar Spann; seine Tätigkeit für die konservative Wochenschrift „,Schönere Zukunft“ als Antikommunist wie als Antikapitalist; die Deportation nach Buchenwald, wo sich ein politischer Wandel vollzog und wo ihm die Tätigkeit als Schreiber für den Lagerarzt Erwin Ding-Schuler wohl das Leben gerettet hat. Leider fehlt als Pendant zum Prolog ein biografischer Epilog. Der Leser erfährt über seine letzten Jahre wenig: Er habe an Depressionen gelitten und sei hoch verschuldet gewesen. Seine einzige Monografie, vom wirkmächtigen ,SS-Staat“ (1946) abgesehen, stammt aus der Zeit nach der Emeritierung (,Die Stunde der Ingenieure“, 1976).

Eckhard Jesse $(\triangle)$

Institut für Politikwissenschaft, TU Chemnitz, Chemnitz, Deutschland

E-Mail: eckhard.jesse@phil.tu-chemnitz.de 
Der Autor hat in der quellengesättigten Studie sein Vorhaben, ein vielschichtiges Porträt Kogons zu präsentieren, alles in allem gut eingelöst. Allerdings kann der Aufbau nicht ganz überzeugen. Die Unterteilung in die 1950er- und die 1960erJahre leuchtet wenig ein, war doch weder für Kogon noch für die Bundesrepublik die Zeit um 1960 eine Zäsur. Beismann unterscheidet in jeder der beiden Phasen zwischen Leben, publizistischem Engagement und Hochschullehrertätigkeit.

Kogon plädierte nach 1945 für einen freiheitlich-christlichen Sozialismus, den er zunächst bei der CDU sah. Die Herausgeberschaft der linkskatholischen „Frankfurter Hefte“ (mit Walter Dirks) von Januar 1946 zählt zu seinen großen Meriten. In mancher Hinsicht Anhänger eines verschwommenen dritten Weges, engagierte er sich als Präsident der Europa-Union (1949-1953) früh in der Europa-Bewegung mit dem Bestreben einer europäischen Föderation als dritter Kraft. Dies war ebenso vergeblich wie sein Kampf gegen die Wiederbewaffnung. All das wird nachvollziehbar erhellt. Fiel die Haltung gegenüber Bundeskanzler Konrad Adenauer immer kritischer aus, zeichnete sich Kogons politische Sichtweise ansonsten durch ein hohes $\mathrm{Maß}$ an Kontinuität aus.

Die Tätigkeit als Hochschullehrer war nicht seine Hauptbeschäftigung, obwohl Kogon der Waldleiniger Konferenz „Einführung der Politischen Wissenschaften an den deutschen Universitäten und Hochschulen“, dem „Startschuss für die Politologie“ (S. 93), im September 1949 beigewohnt und sie mit Redebeiträgen bedacht hatte. Er hielt seine Veranstaltungen ,am Stück“ (S. 105) ab und war bis zur Berufung Arcadius R. L. Gurlands 1962 in Darmstadt der einzige Professor im Fach. Die Lehre an einer Technischen Hochschule muss für den Generalisten ein Glück gewesen sein. Wie Beismann ausführlich belegt, trug maßgeblich Kogon zur Eingliederung der Gewerbelehrerausbildung an der TH Darmstadt bei - so hieß sie bis 1997.

In den 1960er-Jahren nahm die ohnehin schon große Arbeitsbelastung durch die Fülle der ihm angetragenen Ämter weiter zu. Den Job beim Fernsehmagazin „Panorama“ gab der „Fernsehprofessor“ (S. 130) schon nach einem Jahr auf. Kogon, dessen Distanz gegenüber der Politik der Union nach und nach zugenommen hatte, wünschte die SPD in den 1960er-Jahren an die Regierung und unterstützte eine neue Deutschland- wie Ostpolitik. Noch kurz vor der Emeritierung übernahm Kogon das Amt des Vorsitzenden der Deutschen Vereinigung für Politische Wissenschaft. Da die Politikwissenschaft, in der Öffentlichkeit zum Teil verantwortlich gemacht für die Unruhen, seinerzeit in der Defensive war, wollte der Kompromisswillige zwischen den Fronten vermitteln - inner- wie außerhalb des Faches. Auf dem von ihm organisierten Berliner Kongress der Vereinigung 1969 fiel seine Bilanz im Eröffnungsvortrag bei aller Kritik recht positiv aus. Allerdings sind die Passagen mit vielen ellenlangen Zitaten über diese Zeit wenig erhellend. Offenbar kennt sich der Biograf mit den Usancen der geschichtswissenschaftlichen Zunft besser aus als mit denen der politikwissenschaftlichen. Seine These, wonach „Vorwürfe gegen namhafte Politologen“ (S. 203) zum Extremistenbeschluss von 1972 beitrugen, ist kühn, jedenfalls unbelegt.

Bei Kogons Kampf gegen die Notstandsgesetzgebung verweist Beismann auf Aspekte, ,die in keinem direkten Zusammenhang mit dem Streitgegenstand standen“ (S. 183). Der Protest, und damit trifft der Verfasser ins Schwarze, resultierte auch 
aus der „Verfolgungserfahrung“(S. 183). Gleiches gilt für Kogons, vom Autor leider nicht erwähntes, unrühmliches Engagement in Sachen Reichstagsbrand. Er ließ sich von dem hochtrabend sogenannten ,Internationalen Komitee zur wissenschaftlichen Erforschung der Ursachen und Folgen des Zweiten Weltkrieges“, das mit gefälschten Dokumenten die Urheberschaft der Nationalsozialisten am Reichstagsbrand belegen wollte, vor den Karren spannen. Lebensgeschichtlich mag Kogons entsprechendes Engagement verständlich sein, aber unter wissenschaftlichen Gesichtspunkten war es ein Armutszeugnis.

Der Darmstädter, streitbar bis zur Polemik, war ein begabter, ja ein begnadeter Redner, der andere für sich einzunehmen verstand. Allerdings benennt der Biograf immer wieder seine ,autoritäre Ader“ (S. 25, 117, 128, 129, 213), bringt dafür Belege. Kogons Gesammelte Schriften, erschienen zwischen 1995 und 1999, umfassen acht Bände. Die Texte sind gleichwohl eher zurückhaltend ausgewertet worden. Vielleicht war dies gut so, denn seine Autorität geht mehr auf das öffentliche Wirken zurück, weniger auf die Schriften. Beismann überschätzt allerdings dessen Rolle, wenn es heißt, dieser habe, ,in den Schlüsseldebatten der Bundesrepublik [...] zweifellos Einfluss auf den Weg der Bundesrepublik zu einem liberalen Staatsverständnis“ (S. 21) genommen. Er selbst nennt ihn einen ,intellektuellen Exoten“ (S. 214). Die insgesamt flüssig geschriebene Studie gibt einen instruktiven Einblick in das vitale Wirken eines Medienintellektuellen (keines Wissenschafts- und keines Parteiintellektuellen). Wie Beismann nachweist, avancierte der renommierte Publizist Kogon nie zu einem Politikwissenschaftler.

Funding Open Access funding enabled and organized by Projekt DEAL.

Open Access Dieser Artikel wird unter der Creative Commons Namensnennung 4.0 International Lizenz veröffentlicht, welche die Nutzung, Vervielfältigung, Bearbeitung, Verbreitung und Wiedergabe in jeglichem Medium und Format erlaubt, sofern Sie den/die ursprünglichen Autor(en) und die Quelle ordnungsgemäß nennen, einen Link zur Creative Commons Lizenz beifügen und angeben, ob Änderungen vorgenommen wurden.

Die in diesem Artikel enthaltenen Bilder und sonstiges Drittmaterial unterliegen ebenfalls der genannten Creative Commons Lizenz, sofern sich aus der Abbildungslegende nichts anderes ergibt. Sofern das betreffende Material nicht unter der genannten Creative Commons Lizenz steht und die betreffende Handlung nicht nach gesetzlichen Vorschriften erlaubt ist, ist für die oben aufgeführten Weiterverwendungen des Materials die Einwilligung des jeweiligen Rechteinhabers einzuholen.

Weitere Details zur Lizenz entnehmen Sie bitte der Lizenzinformation auf http://creativecommons.org/ licenses/by/4.0/deed.de. 